\title{
Three-Dimensional Reconstruction of a Calyx of Held and Its Postsynaptic Principal Neuron in the Medial Nucleus of the Trapezoid Body
}

\author{
Kurt Sätzler, ${ }^{1,2 *}$ Leander F. Söhl, ${ }^{3 *}$ Johann H. Bollmann, ${ }^{1}$ J. Gerard G. Borst, ${ }^{4}$ Michael Frotscher, ${ }^{3}$ \\ Bert Sakmann, ${ }^{1}$ and Joachim H. R. Lübke ${ }^{3}$ \\ ${ }^{1}$ Department of Cell Physiology, Max Planck Institute for Medical Research, D-69120 Heidelberg, Germany, \\ 2Interdisciplinary Center of Scientific Computing, University of Heidelberg, D-69120 Heidelberg, Germany, ${ }^{3}$ Anatomical \\ Institute, University of Freiburg, D-79104 Freiburg, Germany, and ${ }^{4}$ Department of Neuroscience, Erasmus University \\ Rotterdam, 3015 GE Rotterdam, The Netherlands
}

The three-dimensional morphology of the axosomatic synaptic structures between a calyx of Held and a principal neuron in the medial nucleus of the trapezoid body (MNTB) in the brainstem of young postnatal day 9 rats was reconstructed from serial ultrathin sections. In the apposition zone between the calyx and the principal neuron two types of membrane specializations were identified: synaptic contacts (SCs) with active zones (AZs) and their associated postsynaptic densities (PSDs) constituted $\sim 35 \%$ ( $n=554$ ) of the specializations; the remaining $65 \%(n=$ 1010) were puncta adherentia (PA). Synaptic contacts comprised $\sim 5 \%$ of the apposition area of presynaptic and postsynaptic membranes. A SC had an average area of $0.100 \mu \mathrm{m}^{2}$, and the nearest neighbors were separated, on average, by $0.59 \mu \mathrm{m}$. Approximately one-half of the synaptic vesicles in the calyx were clustered within a distance of $200 \mathrm{~nm}$ of the AZ membrane area, a cluster consisting of $\sim 60$ synaptic vesicles $(n=52$
SCs). Approximately two synaptic vesicles per SC were "anatomically docked."

Comparing the geometry of the synaptic structure with its previously studied functional properties, we find that during a single presynaptic action potential (AP) (1) $\sim 35 \%$ of the AZs release a transmitter quantum, (2) the number of SCs and anatomically docked vesicles is comparable with the low estimates of the readily releasable pool (RRP) of quanta, and (3) the broad distribution of PSD areas [coefficient of variation $(\mathrm{CV})=0.9$ ] is likely to contribute to the large variability of miniature EPSC peaks. The geometry of the reconstructed synapse suggests that each of the hundreds of SCs is likely to contribute independently to the size and rising phase of the EPSC during a single AP.

Key words: electron microscopy; three-dimensional reconstruction; synapse; active zones; puncta adherentia; synaptic vesicles
A quantitative description of the signal cascade underlying synaptic transmission requires that the temporal and spatial dependence of those steps that couple a presynaptic action potential (AP) to a postsynaptic potential must be known. Important steps are (1) the diffusion of $\mathrm{Ca}^{2+}$ in the presynaptic terminal driving the exocytosis of transmitter-containing vesicles (Katz, 1969), (2) the diffusion of transmitter in the synaptic cleft after fusion has occurred, (3) the transient opening of postsynaptic channels after binding of transmitter to its cognate receptors (del Castillo and Katz, 1957), and (4) clearance of transmitter. Some of these steps have been elucidated in the mammalian brain for the glutamatergic giant synapse in the medial nucleus of the trapezoid body (MNTB) in the acoustic pathway. The large somatic terminals of

\footnotetext{
Received Nov. 12, 2001; revised Sept. 23, 2002; accepted Sept. 27, 2002.

We thank S. Nestel for her extraordinary skills in producing large series of ultrathin sections. We thank A. Roth for performing simulations of glutamate diff usion in the synaptic cleft and of AMPAR-mediated EPSCs. We also thank him, together with Drs. T. Kuner, C. Meinrenken, R. Schneggenburger, and J. Waters and Professor E. Neher, for critically reading a previous version of this manuscript. In addition, we thank M. Winter and I. Dehof for excellent technical assistance. Finally, we thank Professor W. Jäger and Drs. R. Eils and K. Saracoglu from the Interdisciplinary Center of Scientific Computing in Heidelberg for technical support and fruitful discussions.

*K.S. and L.F.S. contributed equally to this work.

Correspondence should be addressed to Bert Sakmann, Department of Cell Physiology, Max Planck Institute for Medical Research, Jahnstrasse 29, D-69120 Heidelberg, Germany.

Copyright (C) 2002 Society for Neuroscience $0270-6474 / 02 / 2210567-13 \$ 15.00 / 0$
}

bushy cells in the ventral cochlear nucleus (VCN), known as calyces of Held, form synapses with cell bodies of the principal neurons of the MNTB (Held, 1898; Lenn and Reese, 1966; Nakajima, 1971; Petelina, 1975; Casey and Feldman, 1985; Kandler and Friauf, 1993; Forsythe, 1994; Rowland et al., 2000). The trains of APs in the bushy cells of the VCN are converted to an equivalent inhibitory signal transmitted to the lateral superior olive (LSO) (Smith et al., 1998).

In young rats [postnatal day 9 (P9)] both the presynaptic and postsynaptic elements of the synapse can be patch clamped simultaneously (Borst et al., 1995; Takahashi et al., 1996; Borst and Sakmann, 1998) to measure transmitter release under defined internal and external ionic and membrane potential conditions. The size and time course of AP-evoked calyceal $\mathrm{Ca}^{2+}$ influx (Borst and Sakmann, 1996, 1998), occupancy of the putative $\mathrm{Ca}^{2+}$ sensor driving vesicle fusion (Bollmann et al., 2000; Schneggenburger and Neher, 2000), and the equilibration of intracellular $\mathrm{Ca}^{2+}$ with the endogenous $\mathrm{Ca}^{2+}$ buffer and the eventual $\mathrm{Ca}^{2+}$ clearance (Helmchen et al., 1997) can be measured accurately as well as the latency, size, and time course of evoked quantal EPSCs and multiquantal EPSCs (Borst and Sakmann, 1996; Sahara and Takahashi, 2001).

A quantitative knowledge of the geometry of the presynaptic and postsynaptic structures is required for a more detailed understanding of the mechanisms underlying synaptic transmission and provides important constraints for numerical simulations. On 
the presynaptic side, time- and space-dependent build-up and collapse of $\left[\mathrm{Ca}^{2+}\right]_{\mathrm{i}}$ domains around the internal pore of $\mathrm{Ca}^{2+}$ channels at an active zone (AZ), the buffered diff usion, and the subsequent interaction of free $\mathrm{Ca}^{2+}$ with the $\mathrm{Ca}^{2+}$ sensor can, at present, be simulated only (Yamada and Zucker, 1992; Bertram et al., 1999; Smith, 2001; Meinrenken et al., 2002). Realistic values of the number, distribution, and geometry of synaptic contacts (SCs) including the distribution of synaptic vesicles are essential for constraining such simulations. On the postsynaptic side the time course and amplitude of spontaneous and evoked EPSCs are used to infer the rate of quantal release (del Castillo and Katz, 1954). This inference requires simulations of the transient increase of glutamate concentration in the synaptic cleft, reversible binding of glutamate to glutamate receptor (GluR) channels, and eventual uptake and diffusion of glutamate out of the cleft. To a large extent these processes are governed by the geometry of the synaptic cleft and the postsynaptic densities (PSDs).

Here we report the three-dimensional reconstruction of a giant synaptic structure formed between the calyx of Held and the soma of a principal neuron in the MNTB, made from contours of serial ultrathin sections. The present study provides a quantitative analysis of the number, density, and area of SCs and their associated synaptic vesicles and the volume and geometry of the synaptic cleft.

\section{MATERIALS AND METHODS}

Fixation and tissue processing. All experiments were performed in accordance with the animal welfare guidelines of the University of Freiburg and the Max Planck Society.

For the complete reconstruction of the calyx and the principal neuron, the brain of one Wistar rat was used (selected from four perfused animals, $\mathrm{P} 9$, body weight $\sim 15 \mathrm{gm})$. The animals were anesthetized deeply with sodium pentobarbital (Nembutal, $60 \mathrm{mg} / \mathrm{kg}$ body weight) and perfused transcardially with physiological saline, followed by a phosphatebuffered solution (PB; 0.1 m solution, $\mathrm{pH} 7.4$ ) containing $2.5 \%$ glutaraldehyde (Polyscience Europe GmbH, Eppelheim, Germany) for 15-20 min at room temperature. After $1 \mathrm{hr}$ of postfixation the brains were removed from the skull and stored overnight in fresh fixative at $4^{\circ} \mathrm{C}$. Serial 100- $\mu$ m-thick vibratome sections (Leica VT1000S, Wetzlar, Germany) were cut in the frontal plane through the MNTB complex. After a thorough rinsing in $0.1 \mathrm{M} \mathrm{PB}(3-5 \times, 10 \mathrm{~min}$ for each step), the sections were postfixed for $1 \mathrm{hr}$ in PB-buffered $1 \%$ osmium tetroxide, $\mathrm{pH} 7.4$ (containing $6.86 \mathrm{gm}$ sucrose $/ 100 \mathrm{ml}$ ), at room temperature in the dark. After several rinses $(3-5 \times, 15 \mathrm{~min}$ for each step) in $0.1 \mathrm{M}$ PB the sections were stained en bloc with aqueous $1 \%$ uranyl acetate $(1 \mathrm{hr}$ in the dark). After several rinses they were dehydrated in an ascending series of ethanol (30 min for each step) and propylene oxide (2×, $10 \mathrm{~min}$ for each step) and then flat-embedded in Durcopan (Fluka, Neu-Ulm, Germany). Finally, the sections were polymerized at $60^{\circ} \mathrm{C}$ for $2-3 \mathrm{~d}$.

From one flat-embedded vibratome section through the middle portion of the MNTB, serial ultrathin sections $(\sim 70 \mathrm{~nm}$ in thickness, silver to light gray interference colors) were cut through the MNTB complex on a Reichardt Ultracut S (Leica) and collected on Formvar-coated slot copper grids. Of each of the 270 serial ultrathin sections, four to six electron micrographs of the calyx and the principal cell body were photographed at a primary magnification of $4500 \times$. The micrographs were scanned at high resolution (1800 dpi) on a Zeiss SCAI scanner (Oberkochen, Germany). These images provided the basis for the subsequent three-dimensional morphological reconstruction of the presynaptic and postsynaptic structures.

For the reconstruction of the 52 individual SCs and associated synaptic vesicles, two Wistar rats (P9) were used. One rat brain was perfused transcardially, fixed, and processed according to the protocol described above; the other brain was perfused with a PB-buffered solution containing $1 \%$ paraformaldehyde, $2.5 \%$ glutaraldehyde, and $0.1 \%$ picric acid, $\mathrm{pH}$ 7.4. Vibratome sections of the MNTB complex were processed further according to a modified protocol originally developed by Phend et al. (1992).

Serial ultrathin sections ( $\sim 60 \mathrm{~nm}$ in thickness) through individual synapses were taken from different animals to check for inter-individual differences. Electron microscopic (EM) images were photographed at a primary magnification of $20,400 \times$, and SCs were reconstructed from ultrathin sections taken from both osmium-treated ( $n=32 ; 187$ sections) and osmium-free (Phend et al., 1992) material ( $n=20 ; 136$ sections). No structural differences were found between the two EM preparations, and data sets were pooled. SCs were selected on the basis of their completeness and optimal angle of sectioning, i.e., perpendicular to the synaptic cleft.

Distinction between SCs and puncta adherentia. For the reconstruction of individual SCs $(n=52)$ only those membrane specializations were used that met the following three criteria: (1) presence of synaptic vesicles in close proximity to the presynaptic density, (2) an asymmetry between presynaptic and postsynaptic density, and (3) a widening of the synaptic cleft. For the complete reconstruction all membrane specializations that showed vesicle accumulations and met criterion 1 were classified as SCs. Because of the often nonideal orientation of the synaptic cleft to the plane of sectioning, criteria 2 and 3 applied in only $27 \%$ of all SCs. All other membrane specializations were classified as puncta adherentia (PA). A membrane specialization classified as SC had, on average, $10.6 \pm 8.9$ synaptic vesicles (mean $\pm \mathrm{SD}$ ) located within a distance of $50 \mathrm{~nm}$ to the AZ membrane. In a randomly chosen subset of $n=50 \mathrm{PA}$, we found, on average, $0.6 \pm 1.1$ vesicles within $50 \mathrm{~nm}$ from the membrane specialization. The classification of PA versus SCs agreed among three observers in $>98 \%$ of the cases.

Three-dimensional reconstruction. Three-dimensional reconstructions were made by alignment of serial EM images of the synaptic structure between a single calyx and its principal neuron with the use of a software tool named CAR (Contour Alignment Reconstruction; contact kurt.saetzler@iwr.uni-heidelberg.de)running on a Silicon Graphics computer, as described in detail previously (Sätzler, 2000, 2001). In brief, two successive sections were aligned via rotation and translation such that corresponding structures like mitochondria and membrane specializations in the two sections superimposed. To compensate for distortions introduced by the sectioning, we had to transform the images anisotropically and linearly. Alignment was followed by a contouring of membranes by visually defining markers. The subsequent linear interpolation between these markers resulted in a polygonal membrane "contour." The three-dimensional geometry was reconstructed from the stacks of all contoured sections. By filling these stacks with tetrahedra (Boissonnat, 1988; Boissonnat and Geiger, 1992; Eils and Sätzler, 1999), we calculated volumes and surface areas of the presynaptic and postsynaptic structures of interest.

Data analysis. Surface area values were obtained by summing those triangular areas that formed the boundary of the reconstructed object. Accordingly, the volume of the object was determined as the sum of the volumes of all tetrahedra inside the object (Eils and Sätzler, 1999), which are directly proportional to the estimated section thickness. Varying the average section thickness in the reconstruction by $14 \%$ (assuming a mean thickness of 60 or $80 \mathrm{~nm}$ instead of the $70 \mathrm{~nm}$ used for the reconstruction) leads to the same variation in the reconstructed volume estimate. This represents also an upper bound of the error in surface area measurements introduced by variation of section thickness, although surface measurements are dependent on the real shape and the actual orientation of the reconstructed structure within the slices. For the structure of the reconstructed calyx, varying the section thickness by $14 \%$ results in a variation of the calyx surface area by $5 \%$.

To estimate the number and size of SCs (or PA), we first marked all surface points on the three-dimensional reconstruction of the presynaptic cell membrane that were part of a SC (or PA). In a second step all those triangles of the surface of the calyx were labeled if at least two of the corner points were found to be part of a SC (or PA). In a final step the connected surface patches were defined as follows. Starting from a single, labeled triangle, all those triangles were taken to be connected that met the following criteria: (1) the triangle itself was labeled, and (2) the triangle had at least one side in common with a triangle of the surface patch. Performing this final step iteratively provided individual and connected surface patches defining single SCs (or PA). These surface patches were regrouped manually in part by inspecting the original EM images to compensate for "linking" errors introduced by locally misaligned sections. For each individual patch the surface area and the center of gravity were calculated. We compared this surface area estimate to the product of the summed contour length of a complete $\mathrm{AZ}$ and section thickness (two-dimensional estimate) in a subset of 12 perpendicular cut SCs. The two-dimensional estimate was $17 \%$ lower.

For simulations of nearest-neighbor distances between SCs or between 
PA we used the triangulated mesh of the contact area (see below), attributing contacts randomly to triangles with a probability proportional to their area. Each triangle could contribute to a contact only once. For the simulations we constrained the nearest-neighbor distances to be at least $100 \mathrm{~nm}$ for SCs and PA (similar to the measured values of the reconstructed calyx).

To estimate the cleft volume, we first extracted all those triangles from the reconstructed surface of the calyx and the cell body that were in close apposition to each other $(<70 \mathrm{~nm})$, which we named the contact area. We then estimated the mean cleft width by measuring the mean distance between presynaptic and postsynaptic membranes from the reconstruction ( $28 \pm 9 \mathrm{~nm}$, measured at $n=184,087$ points), which was in good agreement with the cleft width measured directly in sections perpendicularly cut to the synaptic cleft $(27 \pm 4 \mathrm{~nm} ; n=1673)$. The product of mean distance and apposition area provided the estimate of the cleft volume.

The distribution of synaptic vesicles at AZs was determined in individual SCs $(n=52)$. In these contacts we traced the AZ and measured vesicle diameters at the middle of the membrane bilayer. All vesicleto-AZ distances were determined by subtracting the radius of the vesicle from the distance between the center of gravity of each vesicle and the membrane trace of the AZ. This distance denotes a membrane-tomembrane distance.

When the number of docked or clustered vesicles is estimated, the correction factor for possible vesicle double counts according to Abercrombie (1946) would be $\sim 0.56$. Because, however, we counted only those vesicles with a clear ring-like structure, this would cause an underestimate of the true number; therefore, the numbers reported in this study remain uncorrected.

The number of synaptic vesicles clustered at an AZ was determined by counting all vesicles within a $200 \mathrm{~nm}$ distance in sections in which the AZ could be seen. To correct for not including the vesicles located in sections directly adjacent to an AZ, we applied a correction factor of 1.3. This factor represents the ratio between the total intracellular volume within a $200 \mathrm{~nm}$ distance from a (idealized) planar circular AZ (radius, $178 \mathrm{~nm}$ ) and the part of that volume contained in the analyzed sections.

Glutaraldehyde fixation and release. To estimate the amount of vesicular release that might occur during tissue fixation (Smith and Reese, 1980), we measured the presynaptic APs, EPSCs, and kainate-evoked synaptic currents during fixation of an acute MNTB slice preparation. Whole-cell recordings of principal cells and stimulation of afferent fibers were performed as described in detail previously (Borst and Sakmann, 1996). During fixation the afferently evoked EPSCs were abolished (Fig. $1 \mathrm{~A}$, left ) before the presynaptic action potentials or the sensitivity to kainate (Fig. 1 $A$, right) was affected. In addition, the fixation was not accompanied by an increase in the frequency of spontaneous or delayed EPSCs (Fig. $1 B$ ). These results suggest that it is unlikely that the fixative induced a substantial change in the distribution of synaptic vesicles near the presynaptic membrane. The view that negligible vesicular release occurs during fixation also is supported by the fact that we did not observe large numbers of omega-shaped profiles at SCs in the EM images.

\section{RESULTS}

\section{General description and structures of interest}

Figure $2 A$ shows an EM cross section through the synapse between a calyx of Held and an MNTB principal neuron. The image represents a patchwork of five EM micrographs comprising the outlines of the calyx highlighted in yellow and the cell body of the principal neuron in blue, with its nucleus shown in brown. The calyx is a complex nerve terminal forming a cap with fingerlike stalks that envelope the principal neuron. These presynaptic stalks could be followed over several micrometers along the surface of the cell body, sometimes approaching the opposite pole of the principal neuron where they terminate abruptly. The cuplike structure covered $\sim 40 \%$ of the ovoid soma of the principal neuron. The remaining surface area of the principal neuron was not studied in detail; however, some of the finger-like stalks appeared to be surrounded by glial processes. The thickness of the calyx was variable and mostly $<1 \mu \mathrm{m}$. The diameter of the principal cell body was $\sim 22 \mu \mathrm{m}$ in the longer and $18 \mu \mathrm{m}$ in the shorter axis. The nucleus had an eccentric position, being located

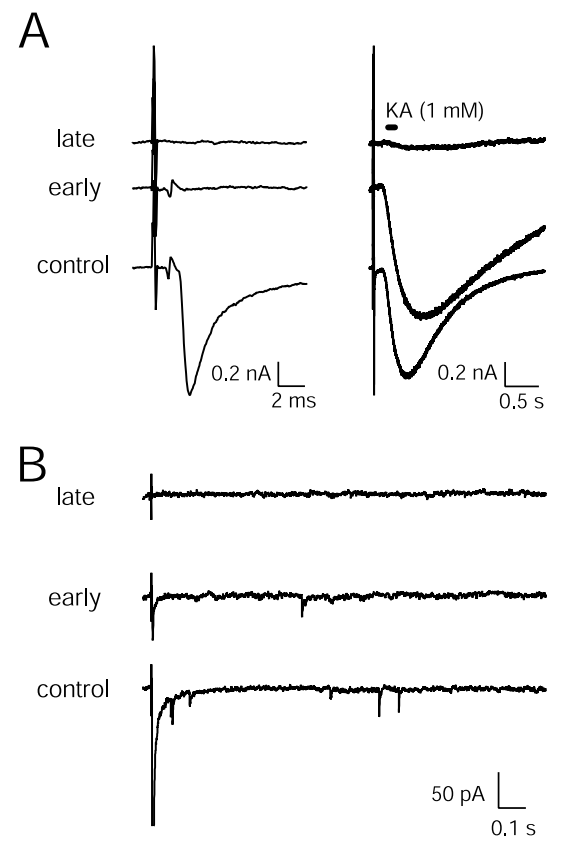

Figure 1. Transmitter release at a MNTB synapse during chemical fixation. A, Left, Afferently evoked prespikes (presynaptic AP recorded in postsynaptic cell) and EPSCs at three different time points during bath perfusion of the experimental chamber with fixatives (1\% paraformaldehyde $/ 2.5 \%$ glutaraldehyde, dissolved in Ringer's solution, $\mathrm{pH} 7.4$ ). At bottom, the control is shown before fixation. The middle traces show $\sim 20$ sec after the start of fixation and the top traces at $\sim 2$ min after the start of fixation. A, Right, The same traces are shown at a lower time resolution, illustrating postsynaptic response to kainate $(K A ; 1 \mathrm{~mm}$, dissolved in Ringer's), which was applied by pressure ejection from a nearby patch pipette. EPSCs were blocked before prespikes or before the sensitivity to KA was clearly changed (middle traces). $B$, Chemical fixation was not accompanied by an increase in spontaneous or delayed EPSCs. In this experiment fixatives were applied by pressure ejection from a nearby pipette. In the bottom trace before fixation the afferently evoked EPSC (amplitude, $\sim 1.5 \mathrm{nA}$ at $-80 \mathrm{mV}$ ) was followed by delayed and spontaneous EPSCs. In the middle trace early during the fixation both evoked EPSCs and spontaneous EPSCs were reduced. In the top trace late during the fixation both evoked and spontaneous EPSCs were blocked. In $A$ and $B$ the traces have been offset vertically for display purposes.

closer to the synaptic membrane. Figure $2 B$ shows the two membrane specializations in the apposition zone between the calyx and postsynaptic membrane (marked region of Fig. $2 A$ at higher magnification), which were classified as SCs (Fig 2, arrow) and PA (Fig. 2, arrowhead), as described in Materials and Methods.

The SCs are spread over the apposition zone between the cell body and the terminal, including the finger-like stalks. They are always characterized by a cluster of synaptic vesicles located in the proximity of the presynaptic density, asymmetric presynaptic and postsynaptic densities, and a broadening of the synaptic cleft (Fig. 3). In their apposition zone the presynaptic and postsynaptic membranes show another membrane structure, which we refer to as PA. These have been described in the adult cat MNTB to be part of an organelle assembly named mitochondria-associated adherens complex (MAC) (Rowland et al., 2000). Figure 4 shows a serial section through a PA.

Because the structure of the calyx changes during development from a cup- to a multifinger-like shape, we measured the mean thickness of the calyx at membrane specializations (MS), compared this value with the mean thickness measured at randomly chosen points, and found that the mean thickness of the calyx at 
Figure 2. Cross section through a calyx of Held and a principal neuron in the MNTB. $A$, EM image of the nerve terminal and the cell body of the principal neuron in the MNTB. The image was obtained by a computer-aided montage of five electron micrographs of a single ultrathin section (imaged at a magnification of $3000 \times$ ). The structures of interest were outlined and laid transparently over the montage; the presynaptic calyx is shown in yellow, the postsynaptic principal neuron in blue, and the nucleus in brown. Scale bar, $5 \mu \mathrm{m}$. $B$, The boxed region in $A$ at higher magnification (imaged at $20,400 \times$ ). The SC is marked by an arrow, and a neighboring PA is marked by an arrowhead. Scale bar, $0.3 \mu \mathrm{m}$.
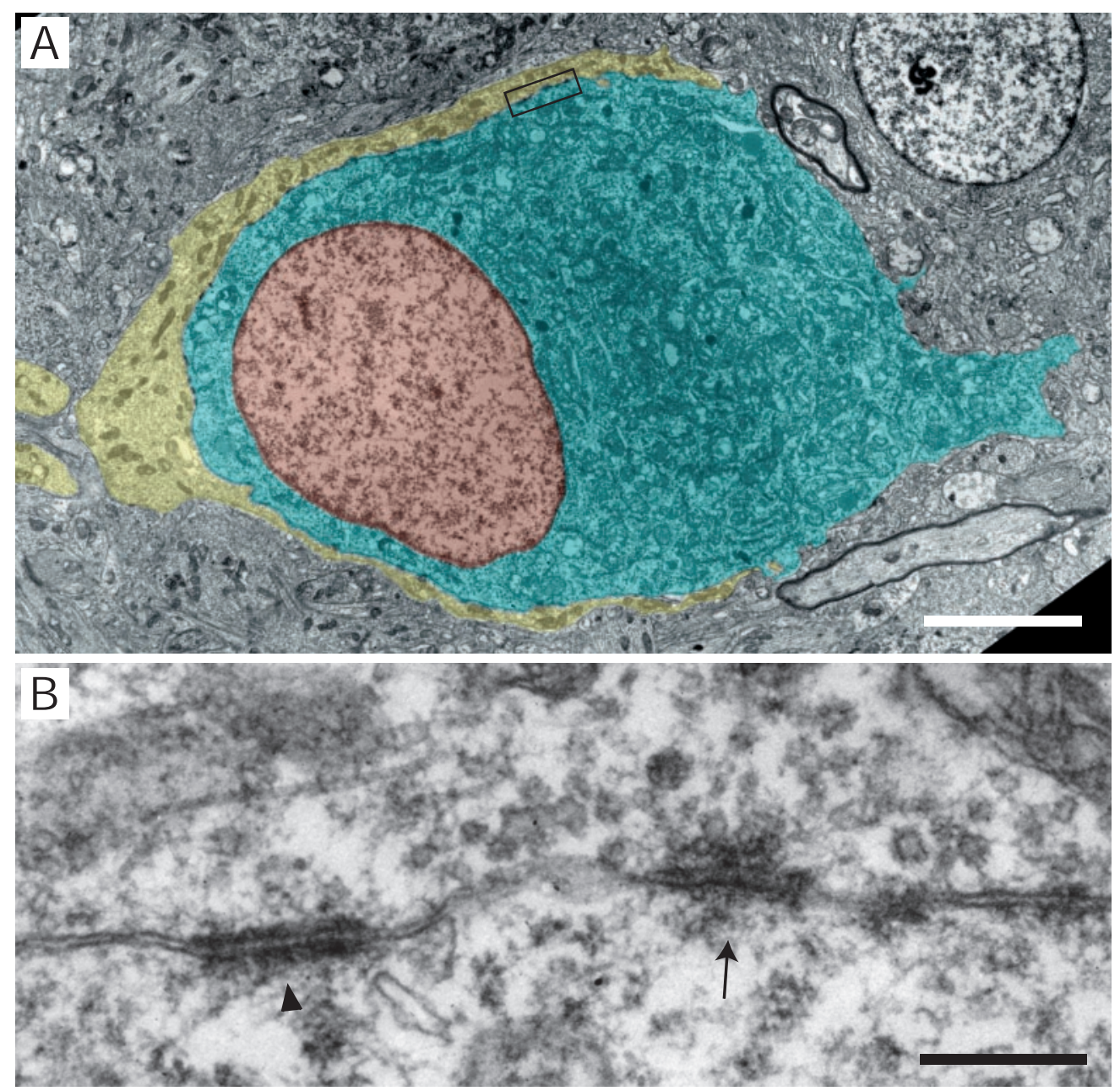

MS is larger than the mean thickness at randomly chosen points. The difference was small but significant (MS, $790 \pm 550 \mathrm{~nm}$; random, $690 \pm 550 \mathrm{~nm}$; Student's $t$ test; $p<0.001)$.

\section{Membrane specializations}

Figures 3 and 4 show serial sections through specialized membrane contacts at high magnification. These were referred to as synaptic contacts (SCs) when synaptic vesicles were accumulated close to the presynaptic dense region (Fig. $3 A-H$ ). The presynaptic and postsynaptic densities formed bands of electron-dense fuzzy material, and both densities were interrupted frequently, appearing as perforated structures. Regularly, dense material connecting the presynaptic and postsynaptic membranes was seen in the synaptic cleft. The SCs shown in Figure $3 A-H$ also exhibited a characteristic widening of the synaptic cleft and an asymmetry in the presynaptic and postsynaptic densities. At some SCs coated vesicles or endocytotic half-vesicles were located at the borders of AZs. Approximately one-third of the membrane specializations in the apposition zone were identified as SCs.

The more frequently occurring type of membrane specialization were PA (Rose et al., 1995). These were characterized by two parallel bands of electron-dense material of approximately the same width at the presynaptic and postsynaptic membranes (Fig. $4 A-H)$ but lacking synaptic vesicles and the wide broadening of the cleft typical for SCs.
In the entire calyx a total of 29 "spine-like" protrusions of the postsynaptic membrane was observed (Fig. 5A,B). They resembled the somatic appendages or somatic spicules described by Tolbert and Morest (1982). In all, 32 SCs were located on these appendages. The protrusions were variable in size, and some of them lacked a spine neck whereas others were comparable with the "stubby" spines on dendrites of neocortical and hippocampal pyramidal neurons, although we never observed a spine apparatus.

Figure 6 shows a three-dimensional reconstruction of a spinelike protrusion with two AZs that are located opposite to each other at the neck. They are relatively large-sized and not perforated. The reconstruction of the volume and surfaces of such structures was made from stacks of contoured sections (Fig. 6A) by filling the stacks with tetrahedra (Fig. 6B).

\section{Geometry of the synapse}

Surfaces of calyx and cell body

Figure 7 shows three-dimensional surface views of the calyx and principal cell body obtained by surface reconstruction from a stack of 270 serial ultrathin sections with a thickness of $\sim 70 \mathrm{~nm}$. The calyx is shown in yellow and the principal neuron in blue. The surface of the calyx and cell body is viewed from two sides by rotating the reconstruction around the horizontal axis (Fig. $7 A, B)$. The calyx rests like a cup on the soma, covering $\sim 40 \%$ of the surface of the principal neuron. From the surface of the calyx 

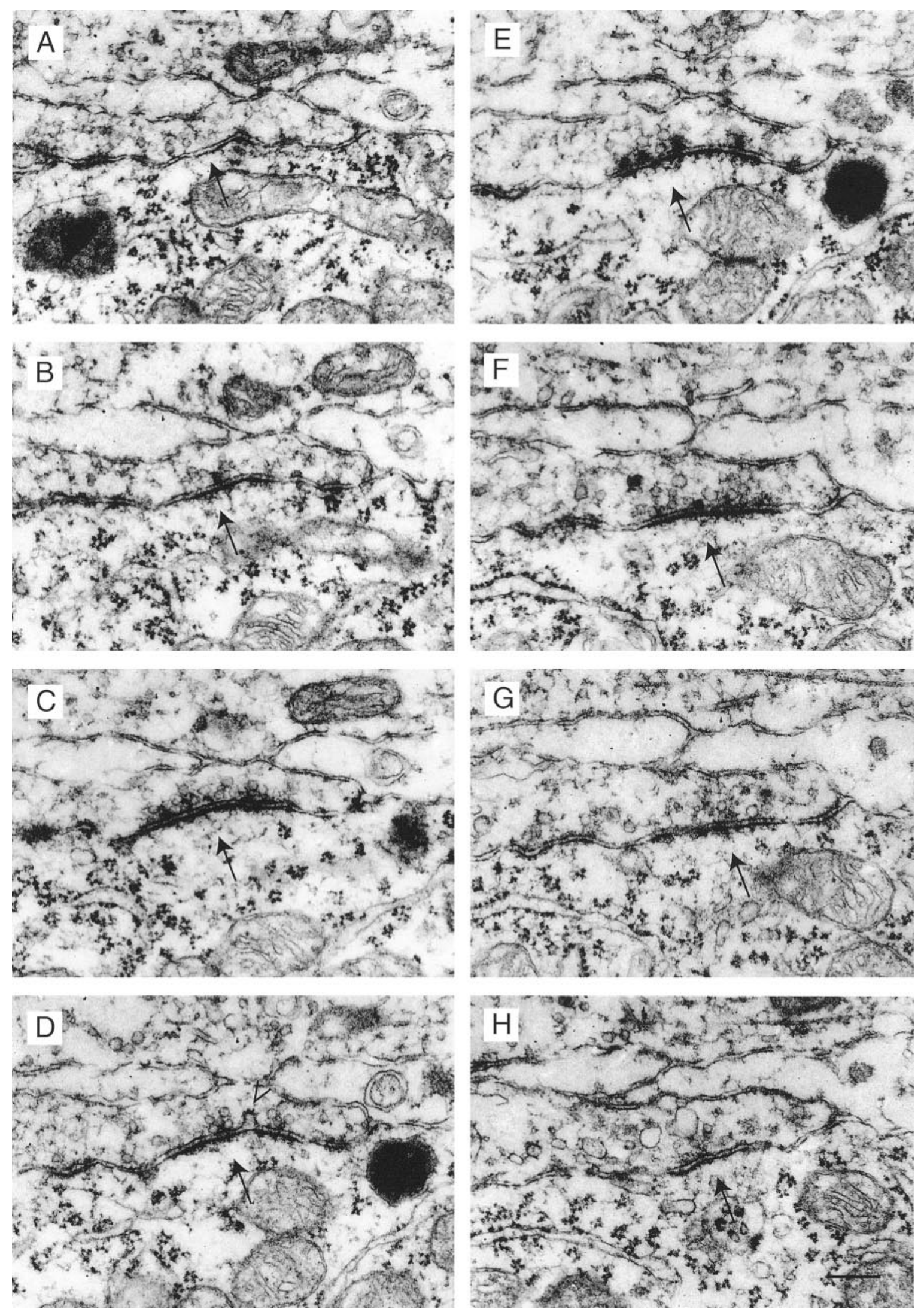

Figure 3. Serial section through a synaptic contact. $A-H$, Selected serial sections of a SC (arrow) between a presynaptic calyx and the postsynaptic principal neuron. The open arrowhead in $D$ points to a vesicle fused with the presynaptic membrane. Scale bar: (in $H) A-H, 0.25 \mu \mathrm{m}$. 

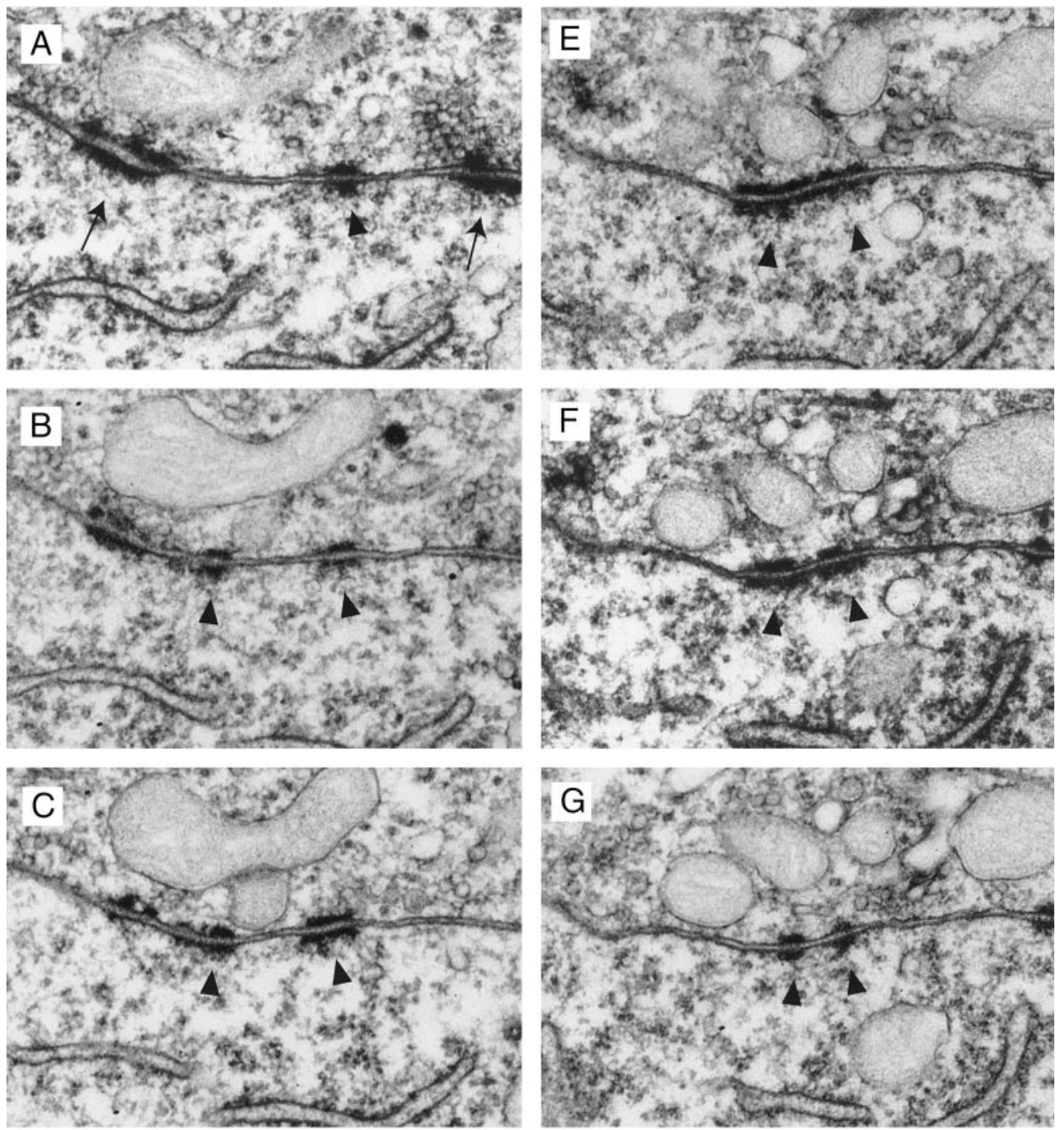

Figure 4. Serial section through a PA $A-H$, Serial sections through a PA are shown. In $A$, two adjacent SCs are visible for a comparison of the two different types of membrane apposition. The PA is labeled by arrowheads, and SCs are labeled by arrows. In contrast to the SCs, the PA shows symmetric presynaptic and postsynaptic densities and lacks a synaptic vesicle accumulation and a widening of the cleft. The whole PA appears to be perforated, as seen in $B-D, G$. Scale bar: (in $H$ ) $A-H, 0.25 \mu \mathrm{m}$.
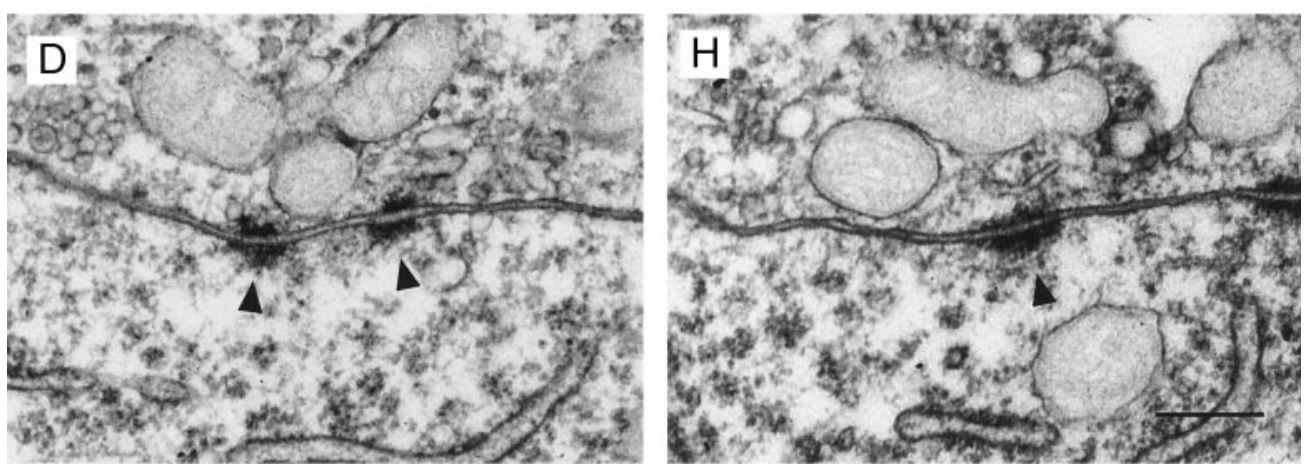

numerous "finger-like" stalks enclose the cell body. In Figure $7 C$ the postsynaptic membrane was made transparent to illustrate the eccentric location of the nucleus, positioned closer to the innervated membrane of the cell body.

\section{Volume and surface area of calyx and soma}

The area of the plasma membrane surrounding the calyx, including both the apposition zone with the principal neuron and the noninnervating part of the membrane, was $\sim 2500 \mu \mathrm{m}^{2}$. The calyceal membrane surrounded a volume of $\sim 480 \mu \mathrm{m}^{3}$ or $0.48 \mathrm{pl}$. The area of the surface membrane of the principal cell body was
$2400 \mu \mathrm{m}^{2}$ of which $\sim 40 \%\left(1000 \mu \mathrm{m}^{2}\right)$ was apposed to the calyx. The volume of the cell body was $\sim 3400 \mu \mathrm{m}^{3}$ or $3.4 \mathrm{pl}$, more than sevenfold larger than the volume of the calyx (Table 1).

\section{Volume of the synaptic cleft}

The volume estimate of the cleft separating the two apposed surface membranes of calyx and soma depends on the degree of "smoothing" of the rugged surface created by triangulation of the contours between successive sections. Distortions of the surface were caused by variations in section thickness (we assumed an 

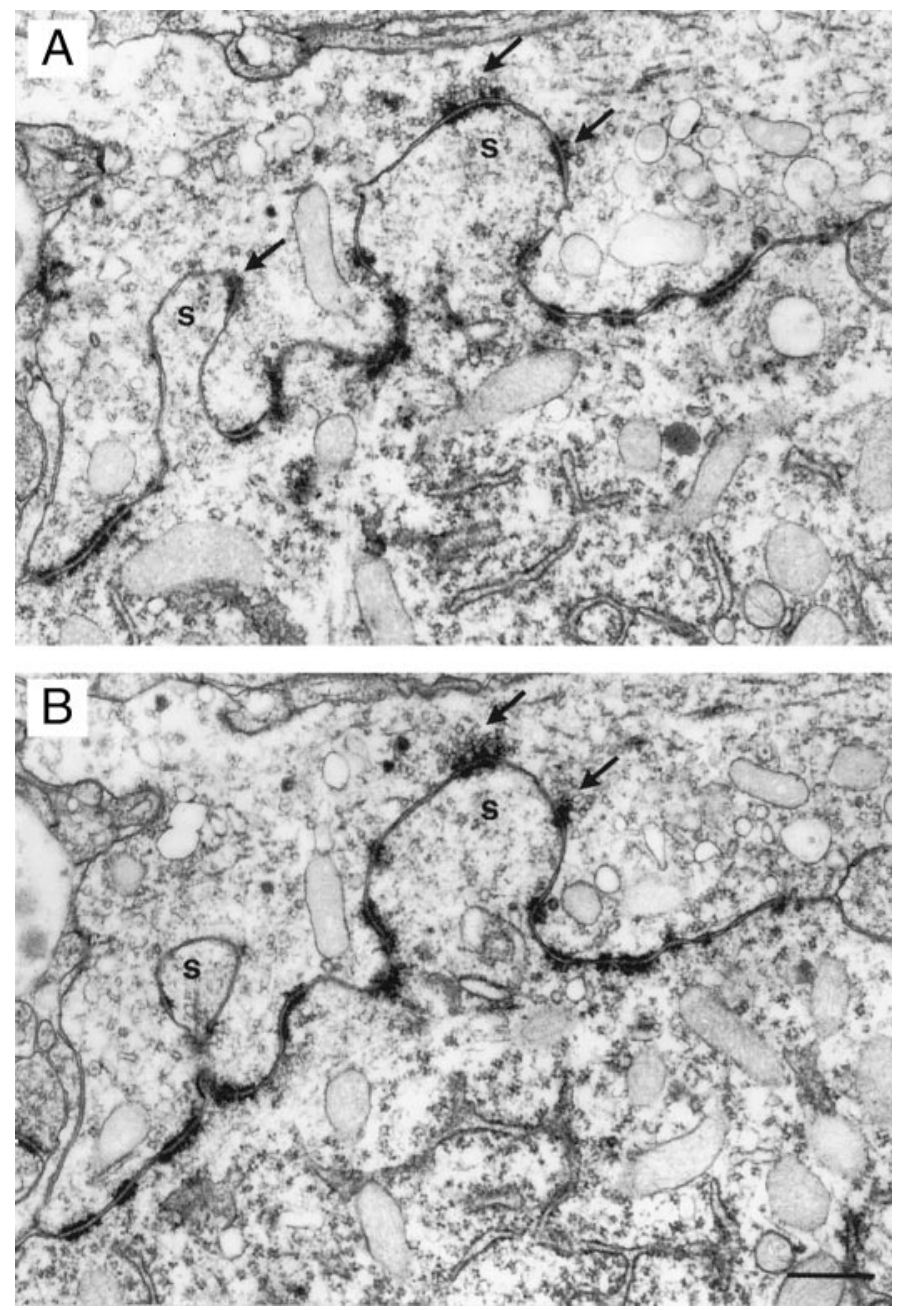

Figure 5. Spine-like protrusion at the soma of the principal neuron. $A, B$, Two successive EM images of sections through two typical spine-like membrane protrusions of the soma of the postsynaptic cell (labeled $s$ ). The protrusion on the left has a narrow "spine neck." Note the presence of SCs identified by the dense accumulation of synaptic vesicles (black arrows) and numerous PA. Only the SCs on spine-like protrusions are labeled. Scale bar, $0.5 \mu \mathrm{m}$.
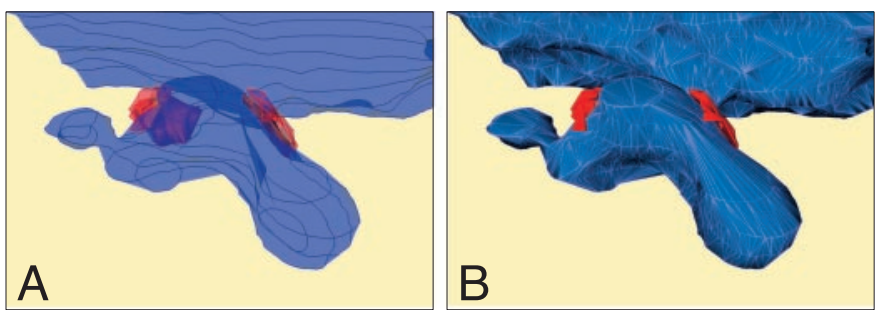

Figure 6. A three-dimensional reconstruction from contoured sections. $A$, Superposition of contoured sections to form a "stack of contours" from serial EM images of a spine-like protrusion. The postsynaptic membrane is marked in blue, the AZs in red, and PSDs in orange. The two SCs are $0.5 \mu \mathrm{m}$ apart. $B$, The stack of contours shown in $A$ was filled by tetrahedra, which generates a triangulated surface (outlines of triangles marked in gray) Section thickness, $60 \mathrm{~nm}$.

average thickness of $70 \mathrm{~nm}$ ) and the sectioning procedure. The distribution of distances measured between the presynaptic and postsynaptic membrane shows that the mean distance was $28 \pm 9$ nm. This value, in combination with the area of the apposition

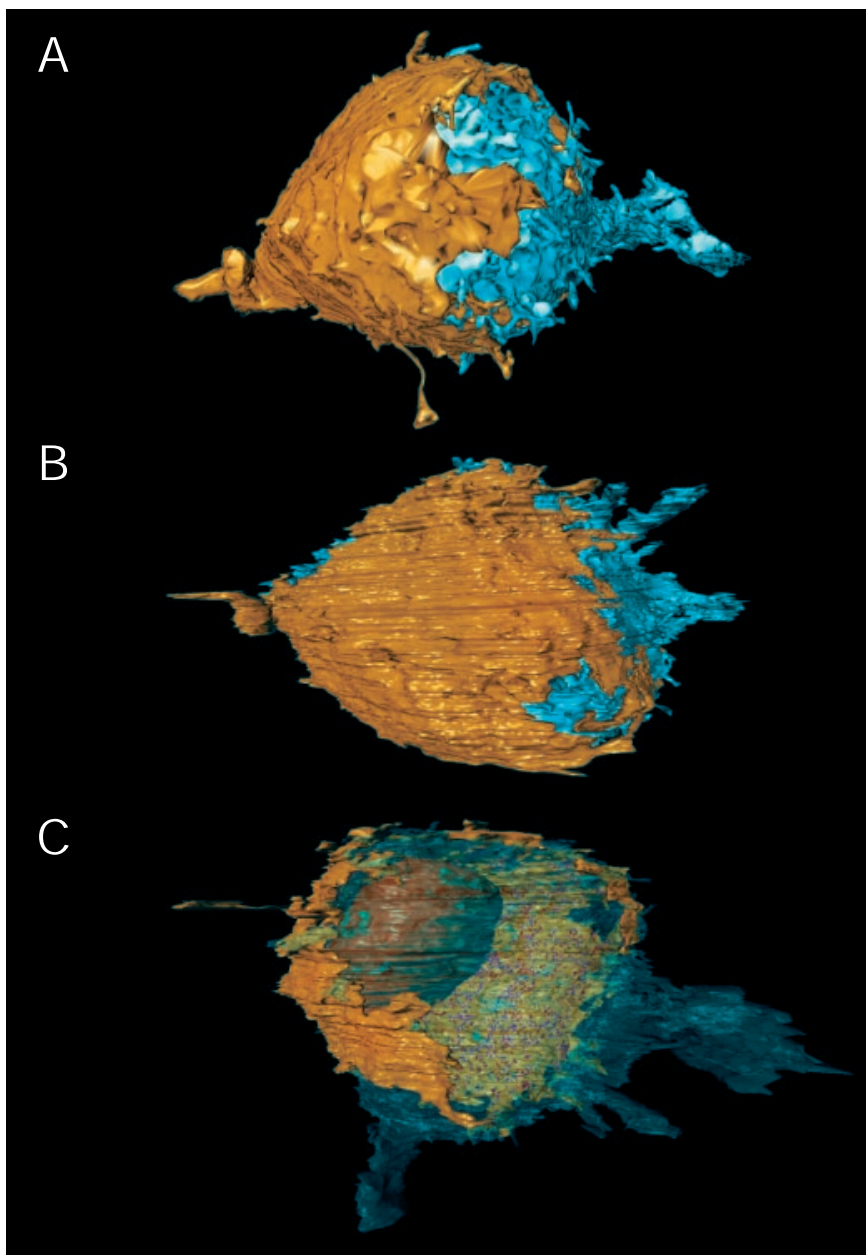

Figure 7. Surface views of the three-dimensional reconstruction of a calyx of Held and the principal neuron. $A$, Top view of the calyx and the cell body of the MNTB principal neuron. The calyx (left) is shown in yellow and the principal neuron (right) in blue. The nerve terminal forms a cup-like structure covering $\sim 40 \%$ of the surface area of the principal neuron. The final part of the preterminal axon that gives rise to the giant terminal is seen on the left. $B$, Side view after rotating the reconstruction by $90^{\circ}$ around the $x$-axis. At the edges of the cap numerous finger-like stalks can be identified. $C$, Surface view of the reconstruction in which part of the surface membrane was made transparent to illustrate the eccentric location of the nucleus (brown) next to the calyx. The membrane specializations can be seen as small spots in red (SCs) and magenta (PA), respectively. The postsynaptic axon (bottom) and dendritic processes (right) were not reconstructed fully.

zone, yields an extrapolated volume of $28.0 \mu \mathrm{m}^{3}$ or $0.028 \mathrm{pl}$ for the synaptic cleft separating presynaptic and postsynaptic cell, $\sim 20$-fold smaller than the volume of the calyx.

\section{Number and size of specialized contacts}

Figure 8 illustrates a view onto the synaptic surface of the calyx. The locations of SCs (Fig. 8A,C, red) and PA (Fig. 8B,C, magenta $)$ are indicated. The number of PA $(n=1010)$ was nearly twofold larger than that of SCs $(n=554)$. The specialized contact zones of the calyx and cell body membrane, not differentiating between SCs and PA, comprised an area of $\sim 100 \mu \mathrm{m}^{2}$, which is $\sim 10 \%$ of the apposition area. Approximately one-half $\left(55 \mu \mathrm{m}^{2}\right)$ of the specialized contact area was occupied by SCs and the other one-half $\left(46 \mu \mathrm{m}^{2}\right)$ by PA, indicating that the SCs were larger, on average. 
Table 1. Geometry of a giant synapse in the MNTB

\begin{tabular}{|c|c|c|c|c|}
\hline & Volume $\left(\mu \mathrm{m}^{3}\right)$ & Surface $\left(\mu \mathrm{m}^{2}\right)$ & & \\
\hline Calyx & 477 & 2484 & & \\
\hline Principal neuron & 3419 & 2443 & & \\
\hline Nucleus & 711 & 526 & & \\
\hline Areas $\left(\mu \mathrm{m}^{2}\right)$ & Number & Total & Mean & $\mathrm{SD}$ \\
\hline Apposition area & & 1022 & & \\
\hline $\mathrm{AZ}$ & 554 & 55.2 & 0.0996 & 0.0861 \\
\hline PA & 1010 & 46.4 & 0.0459 & 0.0419 \\
\hline
\end{tabular}

The mean surface area of a SC was $0.100 \mu \mathrm{m}^{2}$, and its size distribution was characterized by a large variability [coefficient of variation $(\mathrm{CV})=0.9$ ] (Fig. 9A). The surface area of a PA had a mean of $0.046 \mu \mathrm{m}^{2}$, approximately one-half of the size of the area of a SC. Their size distribution shows a similar degree of variability $(\mathrm{CV}=0.9)($ Fig. $9 A)$.

\section{Distance between specialized contacts}

The average distance between nearest-neighbor SCs, measured from their center of gravity, varied widely between 0.15 and 1.3 $\mu \mathrm{m}$, with a mean of $0.59 \mu \mathrm{m}(\mathrm{CV}=0.5)$. The distribution of distances between a PA and its nearest neighbor indicates that PA were, as expected, more closely spaced, the mean nearest-neighbor distance between PA being $0.37 \mu \mathrm{m}(\mathrm{CV}=0.5)$ (Fig. 9B).

To check whether SCs and PA were distributed homogeneously on the apposition zone, we simulated a distribution of points on the calyx membrane that represented the centers of gravity of SCs and PA, respectively. SCs and PA (numbers taken from measurements) were spread randomly across the apposition area. We then compared means and SDs of the distances between nearest-neighbor SCs and PA for the following combinations: distance from SC to closest SC (SC-SC) and closest PA (SC-PA), distance between PA and closest PA (PA-PA) and closest SC (PA-SC), and distance between membrane specializations irrespective of whether they are a SC or a PA (MS-MS). We compared these random distributions with the measured distributions by using a Kolmogorov-Smirnov $(\mathrm{K}-\mathrm{S})$ test to establish whether the simulated and measured distributions of nearest-neighbor distances were different (Press et al., 1992).

The results of the $\mathrm{K}-\mathrm{S}$ test showed that the measured and simulated distributions of nearest-neighbor distances were significantly different for SC-SC distances $(p=0.01)$ and for all other combinations $(p<0.001)$ (Fig. $9 B$, Table 2$)$. This suggests that membrane specializations are not distributed randomly across the apposition area.

\section{Presynaptic active zones}

We attempted to identify subsets of synaptic vesicles as anatomical correlates for different pools of releasable quanta of transmitter as postulated from measurements of release rates (for review, see Schneggenburger et al., 2002). The total number of synaptic vesicles in the calyx and the average number of clustered synaptic vesicles at an AZ were estimated by measuring the number of vesicles located from the AZ within a specified distance of $\sim 500$ and $200 \mathrm{~nm}$, respectively. Furthermore, the number of "anatomically docked" vesicles was estimated by counting

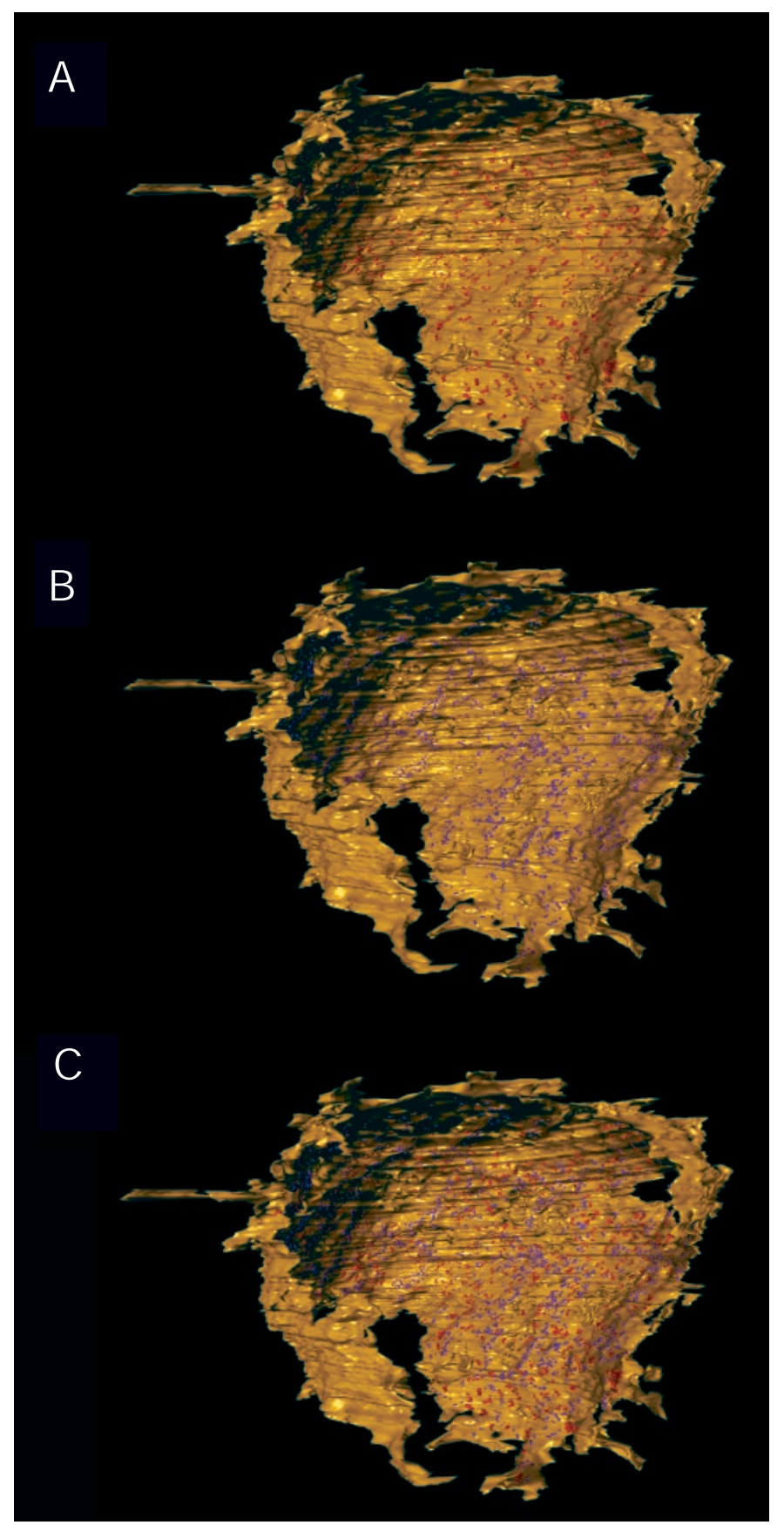

Figure 8. Distribution of membrane specializations in the apposition zone between the calyx and the principal neuron. A, En face view on the synaptic surface of the calyx (yellow) showing the distribution of SCs highlighted in red. $B$, Reconstruction of the same calyx surface as shown in $A$ with the distribution of PA highlighted in magenta. $C$, Same view as in $A$ and $B$ showing an overlay of the distributions of SCs and PA. Same color code is used as in $A$ and $B$.

those vesicles in which no cytoplasmic space was visible between the vesicle and the $\mathrm{AZ}$ membranes (Fig. $10 A-C$ ). A total of 52 SCs was reconstructed, including the vesicles clustered at the AZs. Figure $10 C$ shows an EM image of one representative section through a SC with a cluster of synaptic vesicles located in close proximity of the presynaptic density. The geometric quantities relating to the location and size of synaptic vesicles at an AZ in a section are indicated schematically in Figure $10 D$. 

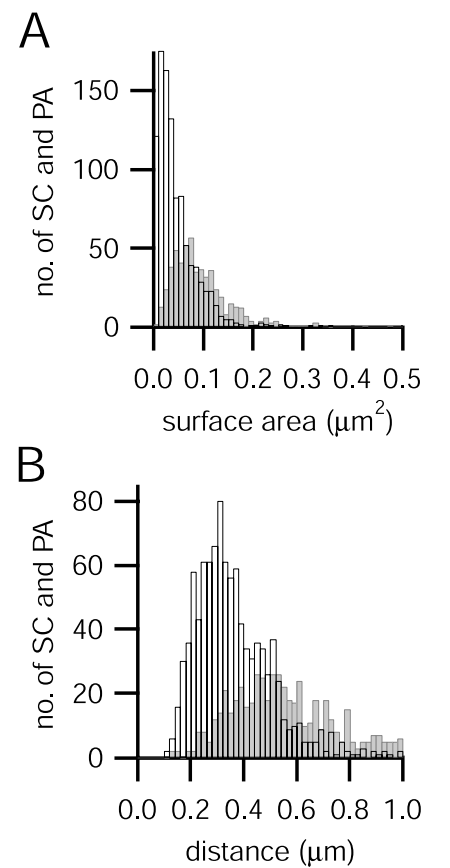

Table 2. Simulation of nearest-neighbor distances between membrane specializations

\begin{tabular}{llllllr} 
& \multicolumn{3}{l}{ Measured } & & \multicolumn{3}{l}{ Simulated } \\
\cline { 2 - 3 } Distances $(\mu \mathrm{m})$ & Mean & SD & & Mean & SD & K-S $p$ \\
\hline SC-SC & 0.590 & 0.296 & & 0.590 & 0.325 & 0.01 \\
SC-PA & 0.489 & 0.214 & & 0.429 & 0.237 & $<0.001$ \\
PA-PA & 0.374 & 0.179 & & 0.444 & 0.229 & $<0.001$ \\
PA-SC & 0.577 & 0.261 & & 0.582 & 0.325 & $<0.001$ \\
MS-MS & 0.364 & 0.146 & & 0.357 & 0.186 & $<0.001$ \\
\hline
\end{tabular}

vesicles of those shown (green) formed a cluster according to our $200 \mathrm{~nm}$ criterion (Fig. 12B), with the average nearest distance from the AZ membrane being $84 \mathrm{~nm}$. The distribution of the number of synaptic vesicles (within a $200 \mathrm{~nm}$ distance cluster) measured for $52 \mathrm{SCs}$ shows that each cluster comprises a mean of $63 \pm 29$ synaptic vesicles (Fig. 12C), which are located at an average distance of $87 \pm 15 \mathrm{~nm}$ from an AZ, corresponding to approximately two vesicle diameters (see below). Extrapolation to $554 \mathrm{SCs}$ and accounting for vesicles located in sections adjacent to the AZ (see Materials and Methods) suggest that in the calyx $\sim 45,000$ synaptic vesicles were clustered around the AZs.

Figure 9. Geometry of membrane specializations in the apposition zones between the calyx and the principal neuron. $A$, Histogram (left) of the distribution of surface areas of SCs ( gray; mean $0.100 \pm 0.086 \mu \mathrm{m}^{2}$ ) and PA (black; mean $0.046 \pm 0.042 \mu \mathrm{m}^{2}$ ) and the corresponding cumulative histogram (right). Bin width, $0.01 \mu \mathrm{m}^{2} . B$, Histogram of the distribution of nearest-neighbor distances between SCs marked in gray (mean, $0.589 \pm$ $0.296 \mu \mathrm{m}$ ) and PA marked in black (mean, $0.374 \pm 0.179 \mu \mathrm{m})$. The right panel shows the corresponding cumulative histograms (same color code). Bin width, $0.02 \mu \mathrm{m}$. The dashed lines are nearest-neighbor distances taken from a simulated representation of SCs (dashed gray line) and PA (dashed black line), which were spread randomly across the apposition area of calyx and principal neuron.

\section{Clusters of synaptic vesicles}

First we determined the number of synaptic vesicles that were found within a certain distance from the membrane of a given SC in all consecutive sections in which the SC could be identified. In the calyx cup-like compartment this distance was $\sim 500 \mathrm{~nm}$, somewhat smaller than the mean thickness of the terminal in this region. Typically, four to five ultrathin sections were needed to reconstruct a SC and to determine the number of associated synaptic vesicles. Figure $11 A$ illustrates a three-dimensional reconstruction of a single $\mathrm{SC}$ in which the number of synaptic vesicles around an AZ could be determined unequivocally. Figure $11 B$ shows two SCs located opposite to each other at a spine-like protrusion with the associated synaptic vesicles, illustrating that, in some cases, distinction between vesicles associated with different SCs is ambiguous. Extrapolating from the sample of $52 \mathrm{SCs}$, with on average $125 \pm 82$ synaptic vesicles, to the total number of AZs $(n=554)$, the calyx would contain at least 70,000 synaptic vesicles (Table 3). More likely, the total number of synaptic vesicles in a calyx is somewhat higher because those located in sections adjacent to SCs and those not associated with SCs were not counted.

Approximately $50 \%$ of the synaptic vesicles were located within a distance of $200 \mathrm{~nm}$ from an AZ (data not shown). Because this distance is approximately one-half of the average distance between nearest-neighbor SCs, synaptic vesicles found within this distance were considered to form a "cluster" associated with the $\mathrm{SC}$, as shown in one example (Fig. 12A). Here, 28 synaptic

\section{Docked synaptic vesicles and vesicle size}

Synaptic vesicles that are docked anatomically (Fig. 10A,B,D, asterisks) to an AZ may represent a morphological correlate of the readily releasable pool (RRP) of quanta as estimated from EPSCs (Schneggenburger et al., 1999; Wu and Borst, 1999) and capacitance measurements (Sun and Wu, 2001). Figure $12 D$ illustrates the distribution of the number of anatomically docked vesicles. The number of docked vesicles increased with $\mathrm{AZ}$ area $\left(r_{\text {Pearson }}=0.62\right.$; data not shown). On average, $2.0 \pm 2.0$ synaptic vesicles were docked per SC ( $n=104$ for $52 \mathrm{SCs}$ ). We also analyzed the average number of synaptic vesicles located within a given distance from an AZ (10-50 nm) (Fig. 12E). The number of synaptic vesicles located within $10 \mathrm{~nm}$ from the AZ membrane $(1.9 \pm 2.0)$ was in good agreement with the number of anatomically docked vesicles. At larger distances from the AZ membrane this number increased from $4.8 \pm 3.8$ (within $20 \mathrm{~nm}$ ) up to $18.1 \pm$ 8.3 (within $50 \mathrm{~nm}$ ).

The diameter of all analyzed synaptic vesicles was $46 \pm 7 \mathrm{~nm}$ (Fig. 12F). We did not observe a significant difference in diameters of synaptic vesicles depending on their location or their distance from the $\mathrm{AZ}$ (data not shown).

\section{DISCUSSION}

Three-dimensional reconstruction of a calyx of Held revealed a structure composed of a cup with several fingers enveloping its postsynaptic target, as previously observed by light microscopy (Kandler and Friauf, 1993). The membrane area $\left(2500 \mu \mathrm{m}^{2}\right)$ and volume $(0.48 \mathrm{pl})$ of the reconstructed calyx corresponded well with estimates derived from measurements of capacitance $(2400$ $\mu \mathrm{m}^{2}$, which includes $<30 \mu \mathrm{m}$ of axon and assumes $10 \mathrm{fF} / \mu \mathrm{m}^{2}$ ) (Borst and Sakmann, 1998) and fluorescence (0.4 pl; Helmchen et al., 1997) of unfixed tissue from animals of the same age (P9). This similarity suggests that tissue fixation and sectioning did not distort overall morphology substantially.

The average surface area of an $\mathrm{AZ}$ was $0.100 \mu \mathrm{m}^{2}$, which lies between the average size of AZs in the end bulb of Held of the juvenile rat $\left(0.066 \mu \mathrm{m}^{2}\right.$; Nicol and Walmsley, 2002) and of the 

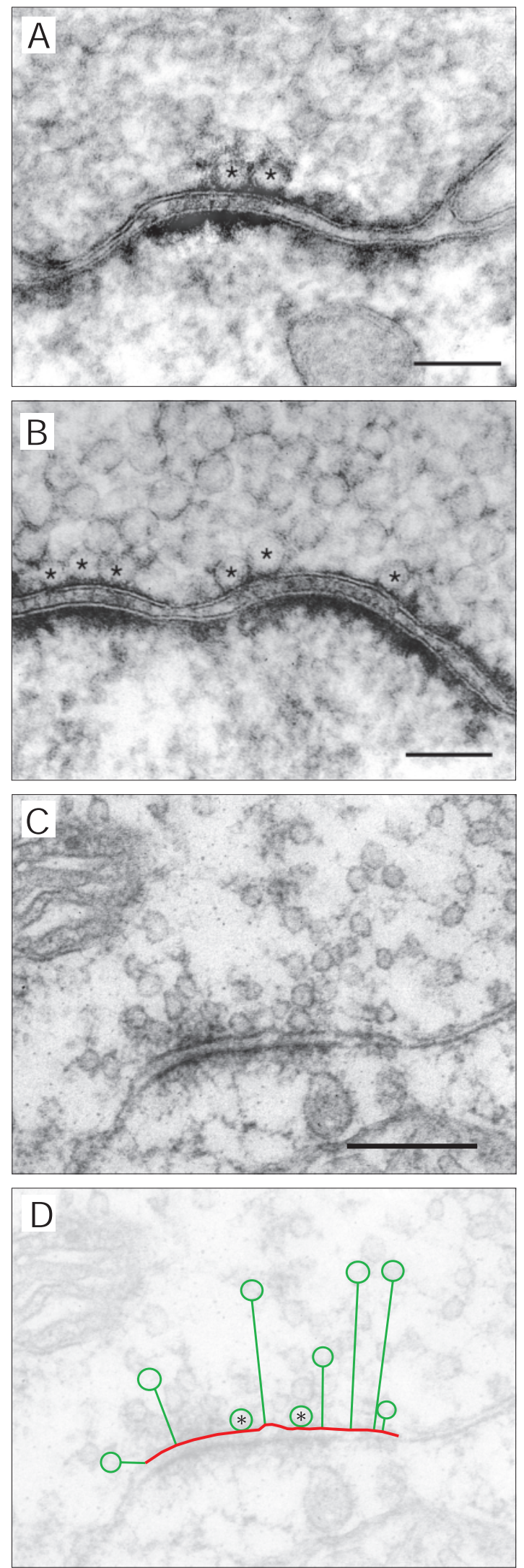

Figure 10. Quantification of synaptic vesicle cluster geometry at SCs. A, $B$, Two different SCs at high magnification with anatomically docked vesicles (marked by asterisks). Scale bar, $0.1 \mu \mathrm{m}$. $C$, Electron micrograph of another single SC showing the dense accumulation of synaptic vesicles at the AZ. Scale bars: $C, D, 0.25 \mu \mathrm{m}$. $D$, Same micrograph as in $C$. Schematic representation of geometric measurements is shown for a
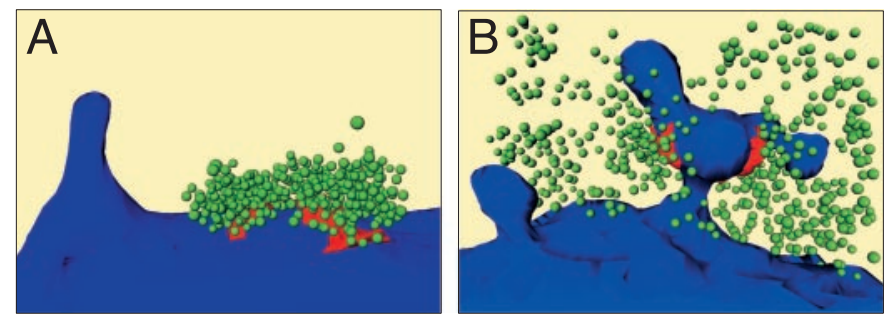

Figure 11. A three-dimensional reconstruction of SCs and associated vesicle clusters. $A$, Cluster of synaptic vesicles near a SC. The surface membrane of the principal neuron is shown in blue, the AZ in red, and synaptic vesicles in green. Note the large size of the pool of synaptic vesicles at this SC $(n=304)$. $B$, Two spine-like protrusions, one with two SCs (on the right). The SCs are located opposite to each other on the neck of the protrusion. Same color code is used as in $A$. Here the individual clusters of synaptic vesicles are not clearly discernible at these two SCs $(n=358)$.

adult cat $\left(0.14 \mu \mathrm{m}^{2}\right.$; Ryugo et al., 1997) and is somewhat larger than that in the adult cat calyx of Held $\left(0.07 \mu \mathrm{m}^{2}\right.$; Rowland et al., 2000). In other mammalian CNS synapses smaller and larger values are observed (range, 0.03-0.14 $\mathrm{mm}^{2}$; Pierce and Mendell, 1993; Harris and Sultan, 1995; Schikorski and Stevens, 1999; Xu-Friedman et al., 2001). In most of these studies a preponderance of small AZs also was observed, suggesting that AZ size distribution is independent of the general morphology of the nerve terminal.

PA are not distributed randomly and may form focal adhesion complexes that stabilize the apposition of presynaptic and postsynaptic membranes. PA also have been reported in the mossy fiber $\rightarrow$ CA3 pyramidal cell synapse (Chicurel and Harris, 1992) and in the CA1 area of the hippocampus (Spacek and Harris, 1998).

\section{Geometry of the calyx of Held as a determinant of release}

A comparison of the number of $\mathrm{Ca}^{2+}$ channels estimated to be open during an AP ( 12,000; Borst and Sakmann, 1996) and the number of SCs in the reconstructed calyx (554) suggests that, for each AZ, $\sim 20$ channels open during the peak of the $\mathrm{Ca}^{2+}$ influx. This value is comparable with the numbers estimated for synaptic boutons in layer 2/3 pyramidal neurons (Koester and Sakmann, 2000). However, not all calcium channels are concentrated at AZs (Wu et al., 1999). A lower estimate is obtained if calcium channels are assumed to be distributed evenly over the entire calyx membrane. In this case only $\sim 0.5$ channels would open per $\mathrm{AZ}$, because the AZ area constitutes $2 \%$ of the total surface area (Table 3). The nearest-neighbor distance between open channels would be $>400 \mathrm{~nm}$. Such low estimates are not in agreement with the apparent low variance in the calcium signals controlling release (Borst and Sakmann, 1999; Meinrenken et al., 2002).

\section{Quantal content of evoked EPSCs and number of SCs}

The estimate of 554 SCs agrees well with the number of functional SCs estimated from fluctuation analysis of evoked EPSCs (Meyer et al., 2001). A single AP releases 200 quanta (Borst and Sakmann, 1996; Schneggenburger et al., 1999; Bollmann et al.,

$\leftarrow$

subset of synaptic vesicles: AZ membrane (red line) and shortest distance (green straight lines) between the membrane of the synaptic vesicles (green circles) and the AZ membrane. Anatomically docked vesicles are marked by asterisks. All parameters were measured in two dimensions. 
Table 3. Geometric quantities of the calyx relevant for release

Measured Extrapolated

Number of AZs

Average area of $\mathrm{AZ}\left(\mu \mathrm{m}^{2}\right)$

554

Average area of synaptic vesicle $\left(\mu \mathrm{m}^{2}\right)$

Number of VDCCs open per AZ, if

VDCCs are distributed in the entire calyx membrane

0.5

VDCCs are located in innervating calyx membrane

VDCCs are located only in AZ membrane

0.0996

0.0067

umber of synaptic vesicles in a calyx $x^{a}$

Number of clustered vesicles within $200 \mathrm{~nm}$ of $\mathrm{AZ}$ in calyx

Number of docked vesicles $(<20 \mathrm{~nm}$ distance) in calyx

Number of docked vesicles $(<10 \mathrm{~nm}$ distance) in calyx
1.25

20

$>70,000$

45,000

2800

1100
${ }^{a}$ The quantity measured at $52 \mathrm{AZs}$ was extrapolated to $554 \mathrm{AZs}$ of the complete reconstruction (mean areas of the two populations of SCs are not significantly different; Student's $t$ test; $p=0.59$ ). VDCC, Voltage-dependent calcium channel.

2000 ), suggesting that only $\sim 35 \%$ of all AZs release a packet of glutamate. Estimates of the size of the readily releasable pool (RRP) of vesicles vary between $\sim 800$ (Schneggenburger et al., 1999; Wu and Borst, 1999; Bollmann et al., 2000) and up to $~ 5000$ (Sun and $\mathrm{Wu}, 2001$ ). The differences are probably attributable to differences in the exact definition of the pool and to the methods used for estimating its size.

The morphological correlate for the RRP of quanta may be the number of anatomically docked vesicles, i.e., those vesicles being in direct contact with the AZ membrane (Schikorski and Stevens, 2001) (but see Xu-Friedman et al., 2001). In the present study the calyx had $<1200$ docked vesicles, in agreement with the lower estimates of the RRP size. If we assume, however, that synaptic vesicles located within $20 \mathrm{~nm}$ from the AZ can be released rapidly, the total number of available synaptic vesicles would be $\sim 3000$, comparable with the midrange estimates of the RRP size (Schneggenburger and Neher, 2000; Sakaba and Neher, 2001). Although more direct experiments are needed to ascertain that vesicles located at $>10 \mathrm{~nm}$ from the membrane can be released within $10 \mathrm{msec}$, the distance from the membrane could contribute to the heterogeneity in their apparent $\mathrm{Ca}^{2+}$ sensitivity (Sakaba and Neher, 2001).

For the largest estimate of the RRP ( $\sim 5000$ releasable vesicles suggested by capacitance measurements) $>8$ vesicles per SC would be released within a few milliseconds. The observed capacitance increase of $\sim 0.4 \mathrm{pF}$ (Sun and $\mathrm{Wu}, 2001$ ) corresponds to an increase in the active zone size of $40 \mu \mathrm{m}^{2}$, almost doubling the AZ area. Under such conditions the diffusional distance between synaptic vesicles and open calcium channels could be increased substantially, which would contribute to the lower release probability of releasable synaptic vesicles during recovery from synaptic depression (Wu and Borst, 1999; Meinrenken et al., 2002). On the other hand, a single AP will lead to an increase in the AZ area of $<10 \%$.

\section{Properties of SCs at other CNS synapses}

Synaptic contacts had, on average, 2.0 docked vesicles, whereas SCs in cerebellum, hippocampus, or cortex contain, on average, at
A

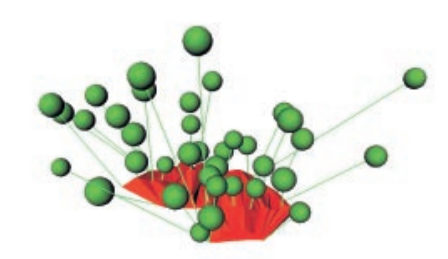

D

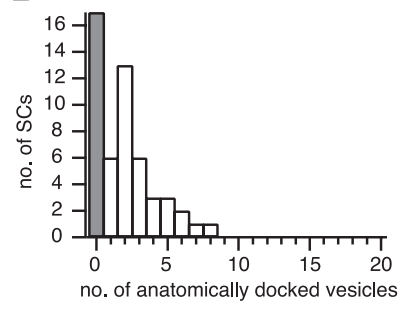

B
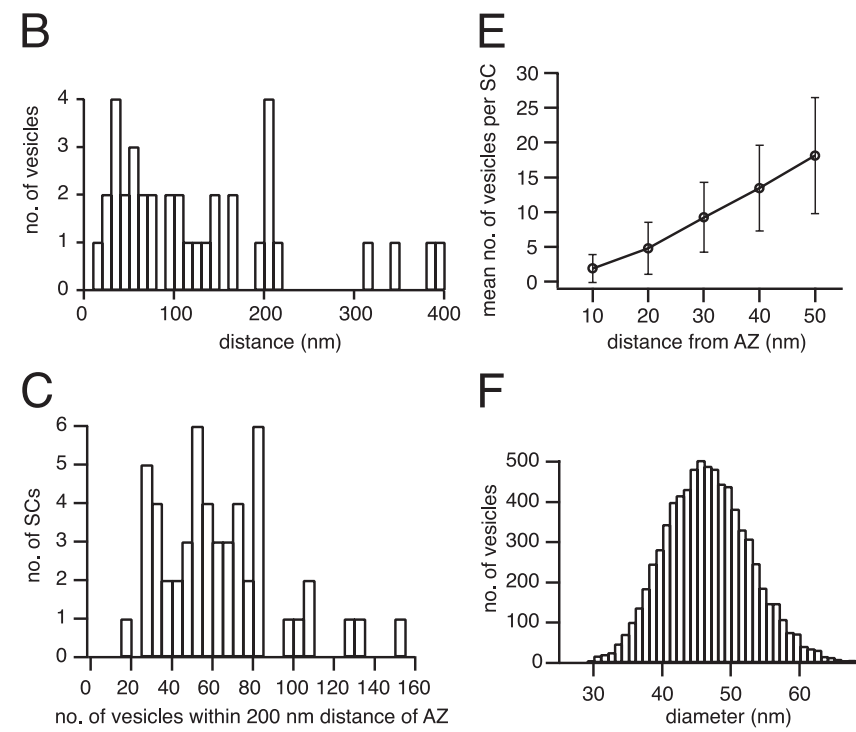

F

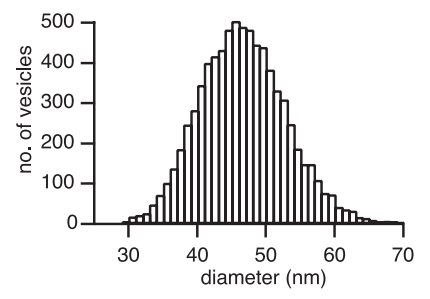

Figure 12. Geometry of synaptic vesicle clusters. $A$, Threedimensional view of a single SC showing the distribution of distances of synaptic vesicles (green) from the AZ membrane (red). Distances are shown as lines connecting a synaptic vesicle and the nearest point on the AZ. For clarity, a relatively small SC was chosen for display. $B$, Histogram of the distribution of distances of synaptic vesicles from the presynaptic membrane at the SC shown in $A$. The total number of synaptic vesicles was 37 . The number of synaptic vesicles clustered within $200 \mathrm{~nm}$ was 28. $C$, Histogram showing the number of SCs with $0-4,5-9$, etc. synaptic vesicles within $200 \mathrm{~nm}$ from the AZ. $D$, Number of SCs with $0,1,2$, etc. anatomically docked vesicles (i.e., no visible cytoplasmic space between synaptic vesicle and AZ membrane). The number of SCs lacking docked vesicles is represented by the gray column. E, Diagram showing the number of synaptic vesicles per SC (mean $\pm \mathrm{SD}$ ) located within a given distance from the $\mathrm{AZ}$ membrane. $F$, Histogram of the distribution of vesicle diameters (mean, $46 \pm 7$ $\mathrm{nm} ; n=7799)$.

least 10 docked vesicles (Harris and Sultan, 1995; Schikorski and Stevens, 1999; Xu-Friedman et al., 2001). The relatively high sensitivity of the release process to the fixatives, i.e., the rapid block of release during superfusion with fixative, argues against a substantial influence of the fixatives on synaptic vesicle distribution in the calyx, in agreement with previous results obtained in cultured neurons (Rosenmund and Stevens, 1997). We cannot exclude that the number of docked vesicles may increase during development, but in the calyx of Held in the adult cat or the end bulb of Held in the juvenile rat these numbers were also comparatively low (Rowland et al., 2000; Nicol and Walmsley, 2002). In most studies, including the present one, the number of docked vesicles varied greatly, with CVs typically ranging between 0.5 and 1.0. This variability may be explained in part by the large variability in AZ sizes, because large AZs generally have more docked vesicles (Schikorski and Stevens, 1999). 


\section{Geometry of PSDs and GluR channels}

The estimated mean number of quanta released per AP ( $\sim 200$; Borst and Sakmann, 1996) indicates that the distance between PSDs activated by released glutamate is $0.9 \mu \mathrm{m}$, on average, assuming random activation of SCs across the apposition zone. Therefore, cross-activation of GluR channels between different SCs is unlikely to shape the rising phase and peak of the EPSC during a single AP-evoked transmission. However, AMPA receptors (AMPARs) desensitize rapidly (Geiger et al., 1995), and simulations of the cleft diffusion of glutamate (A. Roth, unpublished data) indicate that during repetitive stimulation the distance could be short enough to cause GluR activation and/or desensitization by spillover from neighboring release sites (Otis et al., 1996). At experimentally induced maximal release rates this could cause an underestimate of the RRP measured from the EPSC amplitude (Sakaba and Neher, 2001; Sun and Wu, 2001).

\section{Density of GluR channels}

Approximately 20 AMPAR channels open during the peak of a quantal EPSC (Sahara and Takahashi, 2001). Assuming a peak open probability of 0.5 for AMPAR channels at saturating glutamate concentrations (Geiger et al., 1995; Koh et al., 1995), the number of functional channels per PSD would be $\sim 40$. This is a lower limit considering that AMPARs may not be saturated during the release of a single quantum (Silver et al., 1996; Ishikawa et al., 2002). When distributed evenly across the PSD area $\left(0.100 \mu \mathrm{m}^{2}\right)$, the density of functional AMPAR channels would be $\sim 400 \mu \mathrm{m}^{-2}$ with a spacing of $>40 \mathrm{~nm}$. This estimate is comparable with an estimate of the functional AMPAR channel density of PSDs in CA1 and CA3 pyramidal neurons in rat hippocampus (Nusser et al., 1998).

NMDA receptor (NMDAR)-mediated peak current during an evoked EPSC is $\sim 4 \mathrm{nA}$ at $-80 \mathrm{mV}$ in the absence of external magnesium (J. H. Bollmann, unpublished data). If we assume a channel conductance of $\sim 50 \mathrm{pS}$ (Spruston et al., 1995; Clark et al., 1997), then 1000 NMDAR channels are open during the peak of the EPSC, suggesting that approximately five NMDAR channels open at each of the 200 PSDs. Because NMDAR occupancy and open probability depend on the subunit composition (Wyllie et al., 1998), which is not known, these estimates are speculative.

Thus at those PSDs exposed to a transmitter packet, $\sim 20-30$ GluR channels are opened during the peak of the EPSC. The variability $(\mathrm{CV}=0.9)$ of PSD area suggests that the variability in miniature EPSC amplitude (CV of $\sim 0.5$ ) (Borst and Sakmann, 1996; Sahara and Takahashi, 2001) is attributable mostly to the variability in the number of AMPARs present at different SCs.

\section{REFERENCES}

Abercrombie M (1946) Estimation of nuclear population from microtome sections. Anat Rec 94:239-246.

Bertram R, Smith GD, Sherman A (1999) Modeling study of the effects of overlapping $\mathrm{Ca}^{2+}$ microdomains on neurotransmitter release. Biophys J 76:735-750.

Boissonnat JD (1988) Shape reconstruction from planar cross sections. Comput Vis Graph Image Process 44:1-29.

Boissonnat JD, Geiger B (1992) Three-dimensional reconstruction of complex shapes based on the Delaunay triangulation. INRIA Technical Report 1697.

Bollmann JH, Sakmann B, Borst JGG (2000) Calcium sensitivity of glutamate release in a calyx-type terminal. Science 289:953-957.

Borst JGG, Sakmann B (1996) Calcium influx and transmitter release in a fast CNS synapse. Nature 383:431-434.

Borst JGG, Sakmann B (1998) Calcium current during a single action potential in a large presynaptic terminal of the rat brainstem. J Physiol (Lond) 506:143-157.
Borst JGG, Sakmann B (1999) Effect of changes in action potential shape on calcium currents and transmitter release in a calyx-type synapse of the rat auditory brainstem. Philos Trans R Soc Lond B Biol Sci 354:347-355.

Borst JGG, Helmchen F, Sakmann B (1995) Pre- and postsynaptic whole-cell recordings in the medial nucleus of the trapezoid body of the rat. J Physiol (Lond) 489:825-840.

Casey MA, Feldman ML (1985). Aging in the rat medial nucleus of the trapezoid body. II. Electron microscopy. J Comp Neurol 232:401-413.

Chicurel ME, Harris KM (1992) Three-dimensional analysis of the structure and composition of CA3 branched dendritic spines and their synaptic relationships with mossy fiber boutons in the rat hippocampus. J Comp Neurol 325:169-182.

Clark BA, Farrant M, Cull-Candy SG (1997) A direct comparison of the single-channel properties of synaptic and extrasynaptic NMDA receptors. J Neurosci 17:107-116.

del Castillo J, Katz B (1954) Quantal components of the end-plate potential. J Physiol (Lond) 124:560-573.

del Castillo J, Katz B (1957) Interaction at end-plate receptors between different choline derivatives. Proc R Soc Lond B Biol Sci 146:369-381.

Eils R, Sätzler K (1999) Shape reconstruction from volumetric data. In: Handbook of computer vision and applications, Vol 2 (Jähne B, Haussecker H, Geissler P, eds), pp 791-815. San Diego: Academic.

Forsythe ID (1994) Direct patch recording from identified presynaptic terminals mediating glutamatergic EPSCs in the rat CNS in vitro. J Physiol (Lond) 479:381-387.

Geiger JR, Melcher T, Koh DS, Sakmann B, Seeburg PH, Jonas P, Monyer H (1995) Relative abundance of subunit mRNAs determines gating and $\mathrm{Ca}^{2+}$ permeability of AMPA receptors in principal neurons and interneurons in rat CNS. Neuron 15:193-204.

Harris KM, Sultan P (1995) Variation in the number, location, and size of synaptic vesicles provides an anatomical basis for the nonuniform probability of release at hippocampal CA1 synapses. Neuropharmacology 34:1387-1395.

Held H (1898) Die centrale Gehörleitung. Arch Anat Physiol, Anat Abt 201-248.

Helmchen F, Borst JGG, Sakmann B (1997) Calcium dynamics associated with a single action potential in a CNS presynaptic terminal. Biophys J 72:1458-1471.

Ishikawa T, Sahara Y, Takahashi T (2002) A single packet of transmitter does not saturate postsynaptic glutamate receptors. Neuron 34:613-621.

Kandler K, Friauf E (1993) Pre- and postnatal development of efferent connections of the cochlear nucleus in the rat. J Comp Neurol 328: 161-184.

Katz B (1969) The release of neural transmitter substances: the Sherrington lectures. Springfield, IL: Thomas.

Koester HJ, Sakmann B (2000) Calcium dynamics associated with action potentials in single nerve terminals of pyramidal cells in layer $2 / 3$ of the young rat neocortex. J Physiol (Lond) 529:625-646.

Koh DS, Geiger JR, Jonas P, Sakmann B (1995) Ca ${ }^{2+}$-permeable AMPA and NMDA receptor channels in basket cells of rat hippocampal dentate gyrus. J Physiol (Lond) 485:383-402.

Lenn NJ, Reese TS (1966) The fine structure of nerve endings in the nucleus of the trapezoid body and the ventral cochlear nucleus. Am J Anat 118:375-389.

Meinrenken CJ, Borst JGG, Sakmann B (2002) Calcium secretion coupling at calyx of Held governed by nonuniform channel-vesicle topography. J Neurosci 22:1648-1667.

Meyer AC, Neher E, Schneggenburger R (2001) Estimation of quantal size and number of functional active zones at the calyx of Held synapse by nonstationary EPSC variance analysis. J Neurosci 21:7889-7900.

Nakajima Y (1971) Fine structure of the medial nucleus of the trapezoid body of the bat with special reference to two types of synaptic endings. J Cell Biol 50:121-134.

Nicol MJ, Walmsley B (2002) Ultrastructural basis of synaptic transmission between endbulbs of Held and bushy cells in the rat cochlear nucleus. J Physiol (Lond) 539:713-723.

Nusser Z, Lujan R, Laube G, Roberts JD, Molnar E, Somogyi P (1998) Cell type and pathway dependence of synaptic AMPA receptor number and variability in the hippocampus. Neuron 21:545-559.

Otis TS, Wu YC, Trussell LO (1996) Delayed clearance of transmitter and the role of glutamate transporters at synapses with multiple release sites. J Neurosci 16:1634-1644.

Petelina EV (1975) The ultrastructure of synapses in the nucleus of the trapezoid body of the bat. Arkh Anat Gistol Embriol 68:14-18.

Phend KD, Weinberg RJ, Rustioni A (1992) Techniques to optimize post-embedding single and double staining for amino acid neurotransmitters. J Histochem Cytochem 40:1011-1020.

Pierce JP, Mendell LM (1993) Quantitative ultrastructure of Ia boutons in the ventral horn: scaling and positional relationships. J Neurosci 13:4748-4763.

Press WH, Teukolsky SA, Vetterling WT, Flanery BP (1992) Statistical description of data. In: Numerical recipes in C, pp 609-655. Cambridge: Cambridge UP. 
Rose O, Grund C, Reinhardt S, Starzinski-Powitz A, Franke WW (1995) Contactus adherens, a special type of plaque-bearing adhering junction containing M-cadherin, in the granule cell layer of the cerebellar glomerulus. Proc Natl Acad Sci USA 92:6022-6026.

Rosenmund C, Stevens CF (1997) The rate of aldehyde fixation of the exocytotic machinery in cultured hippocampal synapses. J Neurosci Methods 76:1-5.

Rowland KC, Irby NK, Spirou GA (2000) Specialized synapseassociated structures within the calyx of Held. J Neurosci 20:9135-9144.

Ryugo DK, Pongstaporn T, Huchton DM, Niparko JK (1997) Ultrastructural analysis of primary endings in deaf white cats: morphologic alterations in endbulbs of Held. J Comp Neurol 385:230-244.

Sahara Y, Takahashi T (2001) Quantal components of the excitatory postsynaptic currents at a rat central auditory synapse. J Physiol (Lond) 536:189-197.

Sakaba T, Neher E (2001) Quantitative relationship between transmitter release and calcium current at the calyx of Held synapse. J Neurosci 21:462-476.

Sätzler K (2000) 3-D Rekonstruktion von elektronenmikroskopischen Serienschnitten. Naturwissenschaftlich-Mathematische Gesamtfakultät der Universität Heidelberg.

Sätzler K (2001) 3-D Rekonstruktion von Serienschnitten. In: Forschung und wissenschaftliches Rechnen, Beiträge zum Heinz-Billing-Preis 2000 (Plesser T, Hayd H, eds). GWDG-Bericht 56:31-47.

Schikorski T, Stevens CF (1999) Quantitative fine-structural analysis of olfactory cortical synapses. Proc Natl Acad Sci USA 96:4107-4112.

Schikorski T, Stevens CF (2001) Morphological correlates of functionally defined synaptic vesicle populations. Nat Neurosci 4:391-395.

Schneggenburger R, Neher E (2000) Intracellular calcium dependence of transmitter release rates at a fast central synapse. Nature 406:889-893.

Schneggenburger R, Meyer AC, Neher E (1999) Released fraction and total size of a pool of immediately available transmitter quanta at a calyx synapse. Neuron 23:399-409.

Schneggenburger R, Sakaba T, Neher E (2002) Vesicle pools and shortterm synaptic depression: lessons from a large synapse. Trends Neurosci 25:206-212.

Silver RA, Cull-Candy SG, Takahashi T (1996) Non-NMDA glutamate receptor occupancy and open probability at a rat cerebellar synapse with single and multiple release sites. J Physiol (Lond) 494:231-250.
Smith GD (2001) Modeling local and global calcium signals using reaction diff usion equations. In: Computational neuroscience (Schutter ED, ed), pp 49-85. Boca Raton, FL: CRC.

Smith JE, Reese TS (1980) Use of aldehyde fixatives to determine the rate of synaptic transmitter release. J Exp Biol 89:19-29.

Smith PH, Joris PX, Yin TCT (1998) Anatomy and physiology of principal cells of the medial nucleus of the trapezoid body (MNTB) of the cat. J Neurophysiol 79:3127-3142.

Spacek J, Harris KM (1998) Three-dimensional organization of cell adhesion junctions at synapses and dendritic spines in area CA1 of the rat hippocampus. J Comp Neurol 393:58-68.

Spruston N, Jonas P, Sakmann B (1995) Dendritic glutamate receptor channels in rat hippocampal CA3 and CA1 pyramidal neurons. J Physiol (Lond) 482:325-352.

Sun JY, Wu LG (2001) Fast kinetics of exocytosis revealed by simultaneous measurements of presynaptic capacitance and postsynaptic currents at a central synapse. Neuron 30:171-182.

Takahashi T, Forsythe ID, Tsujimoto T, Barnes-Davies M, Onodera K (1996) Presynaptic calcium current modulation by a metabotropic glutamate receptor. Science 274:594-597.

Tolbert LP, Morest DK (1982) The neuronal architecture of the anteroventral cochlear nucleus of the cat in the region of the cochlear nerve root: electron microscopy. Neuroscience 7:3053-3067.

Wu LG, Borst JGG (1999) The reduced release probability of releasable vesicles during recovery from short-term synaptic depression. Neuron 23:821-832.

Wu LG, Westenbroek RE, Borst JGG, Catterall WA, Sakmann B (1999) Calcium channel types with distinct presynaptic localization couple differentially to transmitter release in single calyx-type synapses. J Neurosci 19:726-736.

Wyllie DJA, Behe P, Colquhoun D (1998) Single channel activations and concentration jumps: comparison of recombinant NR1a/NR2A and NR1a/NR2D NMDA receptors. J Physiol (Lond) 510:1-18.

Xu-Friedman MA, Harris KM, Regehr WG (2001) Three-dimensional comparison of ultrastructural characteristics at depressing and facilitating synapses onto cerebellar Purkinje cells. J Neurosci 21:6666-6672.

Yamada WM, Zucker RS (1992) Time course of transmitter release calculated from simulations of a calcium diffusion model. Biophys $\mathbf{J}$ 61:671-682. 